\title{
Th1-mediated experimental autoimmune encephalomyelitis is CXCR3 independent
}

\author{
Stephen J. Lalor and Benjamin M. Segal
}

Department of Neurology, Holtom-Garrett Program in Neuroimmunology, University of Michigan, Ann Arbor, MI, USA

Drugs that block leukocyte trafficking ameliorate multiple sclerosis (MS). Occurrences of opportunistic infection, however, highlight the need for novel drugs that modulate more restricted subsets of $\mathrm{T}$ cells. In this context, chemokines and their receptors are attractive therapeutic targets. CXCR3, a Th1-associated chemokine receptor, is preferentially expressed on $\mathrm{T}$ cells that accumulate in MS lesions and central nervous system (CNS) infiltrates of mice with experimental autoimmune encephalomyelitis (EAE). Surprisingly, mice genetically deficient in either CXCR3 or CXCL10 succumb to EAE following active immunization with myelin antigens. EAE is mediated by a heterogeneous population of $\mathrm{T}$ cells in myelin-immunized mice. Hence, disease might develop in the absence of CXCR3 secondary to the compensatory action of encephalitogenic CCR6 ${ }^{+}$Th17 cells. However, in the current study, we show for the first time that blockade or genetic deficiency of either CXCR3 or of its primary ligand has no impact on clinical EAE induced by the adoptive transfer of highly polarized Th1 effector cells. Our data illustrate the fact that, although highly targeted immunotherapies might have more favorable side effect profiles, they are also more likely to be rendered ineffective by inherent redundancies in chemokine and cytokine networks that arise at sites of neuroinflammation.

Keywords: Autoimmunity - Chemokines - Experimental autoimmune encephalomyelitis • Multiple sclerosis · Th cell

Additional supporting information may be found in the online version of this article at the publisher's web-site

\section{Introduction}

Multiple sclerosis (MS), an inflammatory demyelinating disease of the central nervous system (CNS), is the most common cause of nontraumatic disability among young adults in the United States and Europe. The majority of patients with MS present with a relapsing remitting course, characterized by episodes of neurological disability separated by clinically quiescent periods. Dis-

Correspondence: Dr. Benjamin M. Segal

e-mail: bmsegal@med.umich.edu ease exacerbations correlate with focal breakdown of the bloodbrain barrier and infiltration of the CNS by circulating leukocytes, as reflected by the appearance of gadolinium-enhancing lesions on magnetic resonance imaging (MRI) scans of the brain and spinal cord (SC) [1]. Drugs that block leukocyte trafficking have been shown to ameliorate MS in phase 3 clinical trials. Hence, gadolinium-enhancing lesions and clinical relapses are suppressed by the administration of a mAb specific for the adhesion molecule, $\alpha 4$ integrin, or by treatment with a sphingosine-1-phosphate receptor modulator that prevents the egress of lymphocytes from lymphoid tissues [2,3]. Sphingosine-1-phosphate receptors and $\alpha 4$ integrin are widely expressed on lymphocytes. The introduction of reagents that antagonize those molecules represents a significant 
advance in MS therapeutics. However, there remains a need for novel drugs that modulate more restricted subsets of $\mathrm{T}$ cells in order to maintain clinical efficacy while perturbing protective immunity to the minimum extent possible. In this context, chemokines and their receptors are attractive therapeutic targets for the management of autoimmune disease.

It has long been recognized that the T cells that accumulate in MS lesions are enriched for expression of the chemokine receptor CXCR3 [4-6]. The ELR ${ }^{-}$CXC chemokines, CXCL9 and CXCL10, which are ligands of CXCR3, are expressed by astrocytes and microglia in spatial proximity to perivascular infiltrates $[4,7]$. Similarly, CNS infiltrates of mice with experimental autoimmune encephalomyelitis (EAE, widely used as an animal model of MS) are characterized by a preponderance of $\mathrm{CXCR}^{+} \mathrm{IFN}-\gamma^{+} \mathrm{T}$ cells and upregulation of CXCL10 in adjacent astrocytes [8-11]. These histopathological findings are consistent with the observation that CXCR3 is expressed at high levels on CD4 ${ }^{+}$Th1 cells and can play a critical role in their chemotaxis toward IFN- $\gamma$-inducible $\mathrm{ELR}^{-}$ CXC chemokines [12-14]. Therefore, it was unexpected that mice genetically deficient in CXCR3 or CXCL10 have been shown to be at least as susceptible to EAE as their immunocompetent counterparts [15-17]. Furthermore, in several studies, antagonism of CXCR3 or neutralization of CXCL10 in myelin-immunized wildtype (WT) mice either had no clinical impact or, paradoxically, exacerbated EAE [10,18, 19].

In published studies on the role of CXCR3/ELR ${ }^{-}$CXC chemokines in murine EAE, disease has primarily been induced via active immunization with myelin antigens emulsified in complete Freund's adjuvant (CFA). Mice primed in this manner generate a heterogeneous pool of memory T cells including IFN- $\gamma$ producing Th1 and IL-17-producing Th17 cells [20]. There is also considerable diversity in the cytokine profiles of myelin-specific $\mathrm{T}$ cells isolated from the blood and cerebrospinal fluid of individuals with MS [21,22]. We have previously shown that Th1 and Th17 cells specific for the same myelin epitope induce clinically indistinguishable forms of EAE by invoking the expression of distinct patterns of proinflammatory mediators and chemokines in CNS tissues [23]. Consequently, Th1- and Th17-mediated EAE respond differently to individual immunomodulatory therapies $[21,24,25]$. In addition, there is accumulating evidence that Th1 and Th17 cells employ distinct homing molecules to cross the blood-brain barrier [13, 23, 26]. Therefore, the susceptibility of actively immunized mice to EAE in the absence of functional CXCR3 interactions could be secondary to the compensatory action of encephalitogenic Th17 cells, which have been reported to accumulate in the CNS via a CCR6/CCL20-dependent pathway [26]. We speculated that, under conditions where immune responses are more uniform and highly polarized, the relative importance of CXCR3/CXC chemokine interactions might vary based on the Th bias of the peripheral autoreactive T-cell repertoire. In the current study, we used an adoptive transfer EAE model to investigate whether CXCR3 and/or its ligands are viable therapeutic targets for the treatment of inflammatory demyelinating disease mediated by a Th1-skewed effector cell population.

\section{Results}

\section{$\mathrm{CXCR3}^{-/-}$and $\mathrm{CXCL} 10^{-/-}$mice are susceptible to MOG induced EAE}

Consistent with previous reports [15-17], we found that $\mathrm{CXCR}^{-/-}$and $\mathrm{CXCL}^{-/-}$mice on a C57BL/ 6 background readily succumb to EAE induced by active immunization with myelin oligodendrocyte glycoprotein (MOG) ${ }_{35-55}$ in CFA. Furthermore, disease incidence, the clinical course, and degree of CNS infiltration did not differ significantly between knockout mice and their WT counterparts (Fig. 1A, B, E, and F). Splenocytes and draining LN (dLN) cells harvested from MOGimmunized WT, $\mathrm{CXCR}^{-/-}$and $\mathrm{CXCL} 10^{-/-}$mice mounted comparable IFN- $\gamma$ and IL-17 recall responses upon antigenic challenge ex vivo (Fig. 1C and G). Interactions between CXCR3 and its ligands (CXCL9, CXCL10, and CXCL11) can play a critical role in the chemotaxis of Th1 cells to the CNS [27]. Paradoxically, IFN- $\gamma$-producing cells were more prevalent than IL-17producing cells in CNS mononuclear fractions from $\mathrm{CXCR}^{-/-}$and $\mathrm{CXCL}^{-/-}$, as well as WT, mice with MOG-induced EAE (Fig. 1D and $\mathrm{H})$.

Enrichment of IFN- $\gamma$ producers within the CNS could be secondary to preferential trafficking, survival, and/or expansion of Th1 over Th17 cells. If so, the data in Figure 1 would insinuate that MOG-specific Th1 cells cross the blood-brain barrier and are retained in the brain and SC by a CXCR3/CXCL10-independent mechanism. Alternatively, the majority of CNS-infiltrating IFN- $\gamma$-producing $\mathrm{T}$ cells could represent transformed Th17 cells that acquire Th1-like characteristics within the CNS microenvironment (the so-called "ex-Th17" cells) [28]. Th17 cells have been shown to access the CNS via a CCR6-CCL20-dependent pathway, which could explain the dispensability of CXCR3-CXC chemokine interactions for the development of IFN- $\gamma$-rich neuroinflammatory infiltrates in MOG-immunized mice [26]. In support of the latter hypothesis, mRNA for IL-17A, ROR $\gamma \mathrm{t}$, and CCL20 was upregulated in the CNS of $\mathrm{CXCR}^{-/-}$, CXCL10 $0^{-/-}$, and WT mice with EAE (Fig. 1I and J).

\section{CXCR3 signaling is dispensable for the development and function of encephalitogenic Th1 cells}

Next, we sought to directly compare the relative dependence of MOG-specific Th1 and Th17 cells on CXCR3/ELR ${ }^{-}$CXC chemokine interactions for their encephalitogenicity. MOG-primed CXCR3 ${ }^{-/-}$ T cells exhibited similar cytokine profiles to their WT counterparts following culture under either Th1- or Th17-polarizing conditions (Fig. 2A). As expected, MOG-primed, IL-23-polarized CXCR3 ${ }^{-/-}$ Th17 cells were as efficient as WT Th17 cells in trafficking to the CNS and inducing clinical EAE following adoptive transfer into naïve syngeneic WT hosts (Supporting Information Fig. 1 and Fig. 2B). Surprisingly, IL-12-polarized CXCR3 ${ }^{-/-}$Th1 cells showed no defect in EAE induction (Fig. 2C). In fact, recipients 

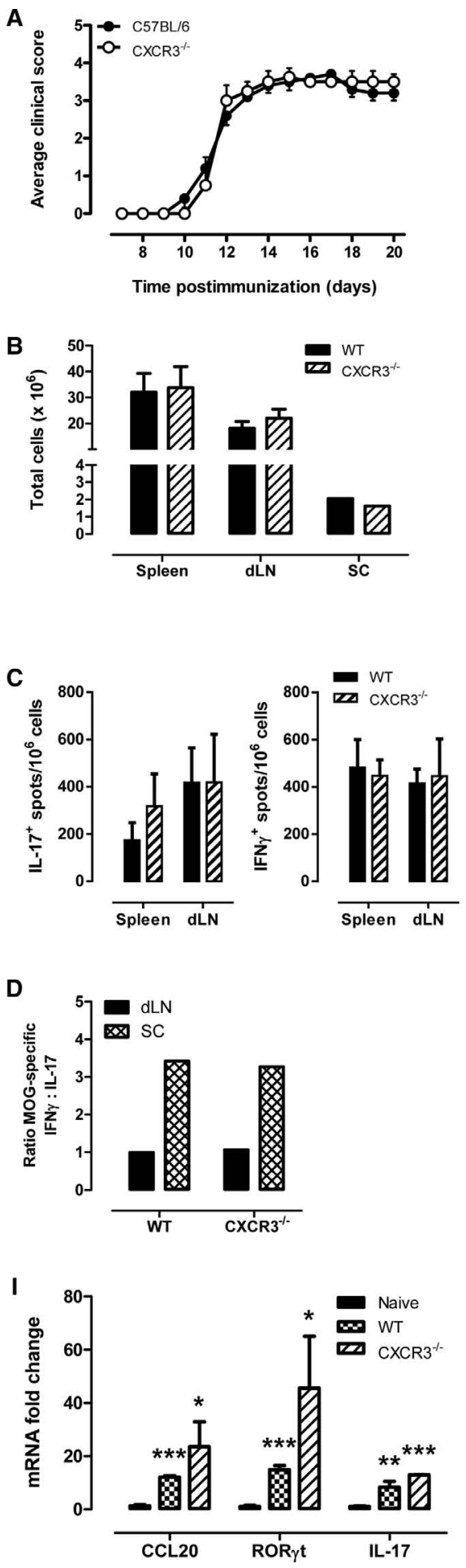
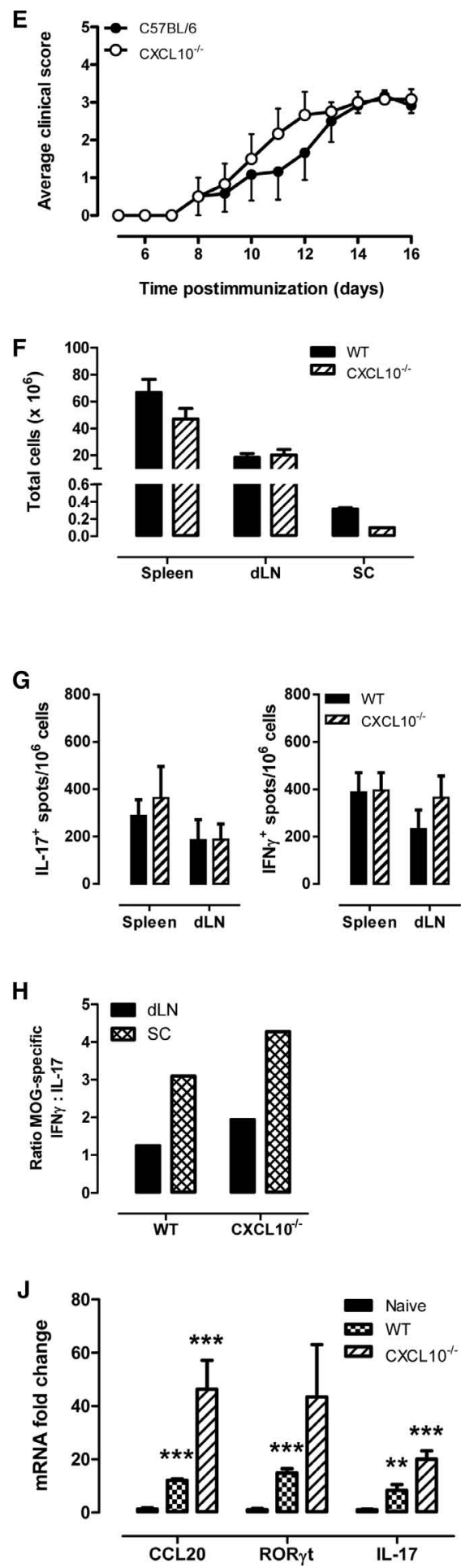

Figure 1. $\mathrm{CXCR}^{-/-}$and CXCL10 ${ }^{-/-}$mice are susceptible to MOG-induced EAE. WT C57BL/6 and (A) CXCR3 ${ }^{-/-}$or (E) CXCL10 ${ }^{-/-}$mice were actively immunized with $\mathrm{MOG}_{35-55}$ in CFA and rated for clinical signs of EAE on a daily basis. (B and F) Spleens, draining lymph nodes (dLNs), and spinal cords (SC) were harvested at (B) day 20 and (F) day 16 postimmunization. The total number of live cells per tissue was counted by Trypan blue exclusion assay. Splenocyte suspensions were subjected to red blood cell lysis prior to counting. SC mononuclear cells were pooled from each group ( $n=4-6$ mice per group) and isolated over a Percoll gradient. ( $C$ and $\mathrm{G}$ ) Cell suspensions were cultured with or without 50 $\mu$ g/mL MOG $35-55$ for ELISPOT assay. Background spots (that developed without the addition of antigen) were subtracted to generate the data shown. Data represent mean +SEM. (D and H) The ratio of MOG-specific IFN- $\gamma$ producers over MOG-specific IL-17 producers is shown for the indicated tissues. (I, J) In separate experiments, SCs from MOG $35-55$-immunized WT C57BL/6 or (I) CXCR3 ${ }^{-/-}$or (J) CXCL10 ${ }^{-/-}$mice were analyzed by real-time RT-PCR. Data represent fold induction relative to naive SCs. (A-J) Data are shown as mean +SEM of four to six mice per group from one experiment representative of three performed. ${ }^{*} p<0.05 ;{ }^{* *} p<0.01 ;{ }^{* * *} p<0.001$, compared with naïve cords, unpaired Student's t-test. 
A

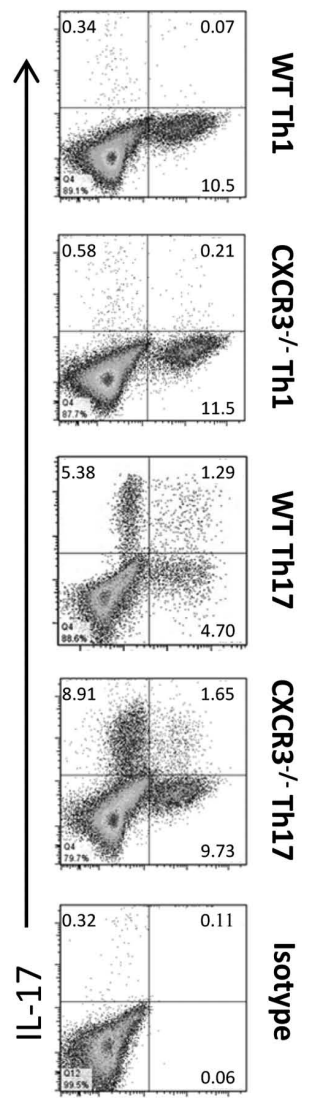

IFN- $\gamma$
B

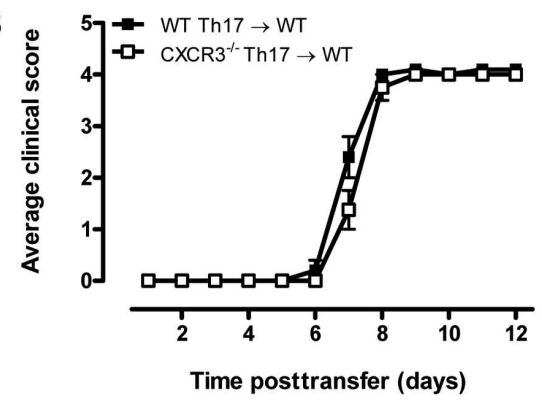

C

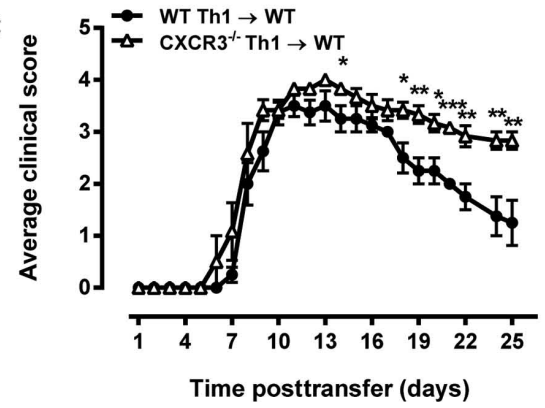

D

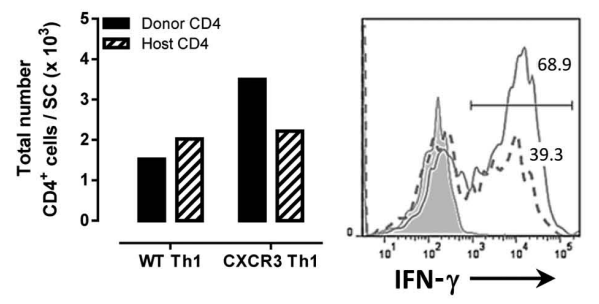

E
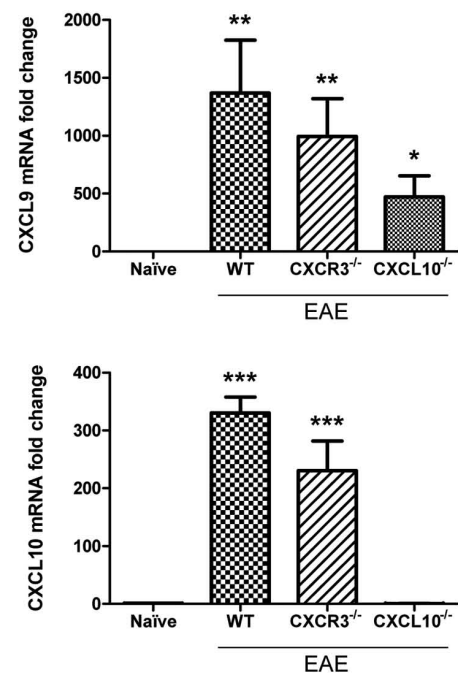

F

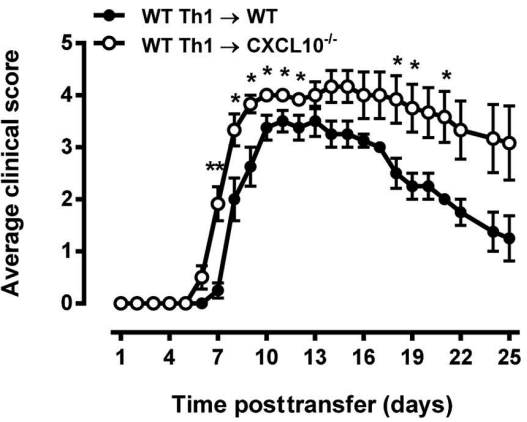

Figure 2. CXCR3 signaling is dispensible for the development and function of encephalitogenic Th1 cells. Ten days after immunization with MOG $_{35-55}$ in CFA, draining LN cells were harvested from WT C57BL/6 or CXCR3 ${ }^{-1-}$ mice and cultured under conditions favorable to the generation of Th1 or Th17 cells. (A) Prior to transfer, $\mathrm{CD}^{+} \mathrm{T}$ cells were analyzed for intracellular cytokine expression following mitogenic stimulation. (B) $2 \times 10^{6}$ WT or CXCR3 ${ }^{-1-}$ Th17 cells were injected i.p. into naïve WT C57BL/6 hosts, which were monitored for clinical signs of EAE on a daily basis. Data are shown as mean \pm SEM of $n=5$ mice per group from one experiment representative of two independent experiments performed. (C) $2 \times 10^{6}$ WT or CXCR3 $3^{-1-}$ Th1 cells were injected i.p. into naïve congenic CD45.1 ${ }^{+}$WT C57BL/6 hosts and monitored for clinical signs of EAE on a daily basis. Data are shown as mean \pm SEM of $n=4-6$ mice per group from one experiment representative of three independent experiments performed. ${ }^{*} p<0.05 ;{ }^{* *} p<0.01 ;{ }^{* * *} p<0.001$, compared with recipients of WT Th1 cells, two-way ANOVA with repeated measures test. (D) At the end of the experiment, spinal cord (SC) mononuclear cells were isolated over a Percoll gradient, pooled and analyzed by flow cytometry. The absolute numbers of $\mathrm{CD} 45.2^{+}$donor cells and CD45.1+ congenic host cells were determined per SC. The histogram shows intracellular IFN- $\gamma$ expression by the donor cells in each group (broken line, WT; solid line, CXCR3 ${ }^{-1-}$ ). (E) WT C57BL/6 or CXCR3 ${ }^{-1-}$ or CXCL10 ${ }^{-1-}$ mice were immunized with MOG $_{35-55}$ in CFA and injected with PT. SCs harvested at the peak of disease were analyzed by real-time RT-PCR. Data represent fold induction relative to naive SCs and are shown as mean + SEM of $n=3-5$ mice per group from one experiment representative of two performed. ${ }^{*} p<0.05$; ${ }^{* *} p<0.01 ;{ }^{* * *} p<0.001$, compared with naïve cords, unpaired Student's t-test. (F) $2 \times 10^{6}$ WT Th1 cells were injected i.p. into naïve WT C57BL/6 or CXCL10 $10^{-/-}$host mice. Hosts were monitored for clinical signs of EAE on a daily basis. Data are shown as mean \pm SEM of $n=4-6$ mice per group from one experiment representative of three performed. ${ }^{*} p<0.05 ;{ }^{* *} p<0.01 ;{ }^{* * *} p<0.001$, compared with WT hosts, two-way ANOVA with repeated measures test.

of $\mathrm{CXCR}^{-/-}$Th1 cells underwent a prolonged disease course with attenuated remission compared to recipients of WT Th1 cells. CXCR3 ${ }^{-/-}$Th1 cells accumulated in the CNS in comparable numbers to WT Th1 donor cells, and the majority of CXCR3 ${ }^{-1-}$ donor T cells in the SC were IFN- $\gamma^{+}$(Fig. 2D). CXCL10 is a dominant CXCR3 ligand in the CNS of the EAE models employed in our studies; C57BL/6 mice do not produce functional CXCL11 protein and CXCL10 is significantly upregulated in the inflamed CNS of MOG-immunized mice (Fig. 2E). In parallel experiments, $\mathrm{CXCL}^{-1} 0^{-/}$and WT hosts exhibited a comparable degree of susceptibility to EAE mediated by WT Th1-polarized, MOG-specific effector T cells (Fig. 2F). Similar to WT recipients of CXCR3 ${ }^{-/-}$ Th1 cells, CXCL10 ${ }^{-/-}$recipients of WT Th1 cells experienced a relatively severe disease course.

\section{CXCR3 blockade does not attenuate Th1-mediated EAE}

Mice that are genetically deficient in an immunological molecule can develop compensatory pathways as they mature. In order to investigate the consequence of disrupting CXCR3 interactions in 

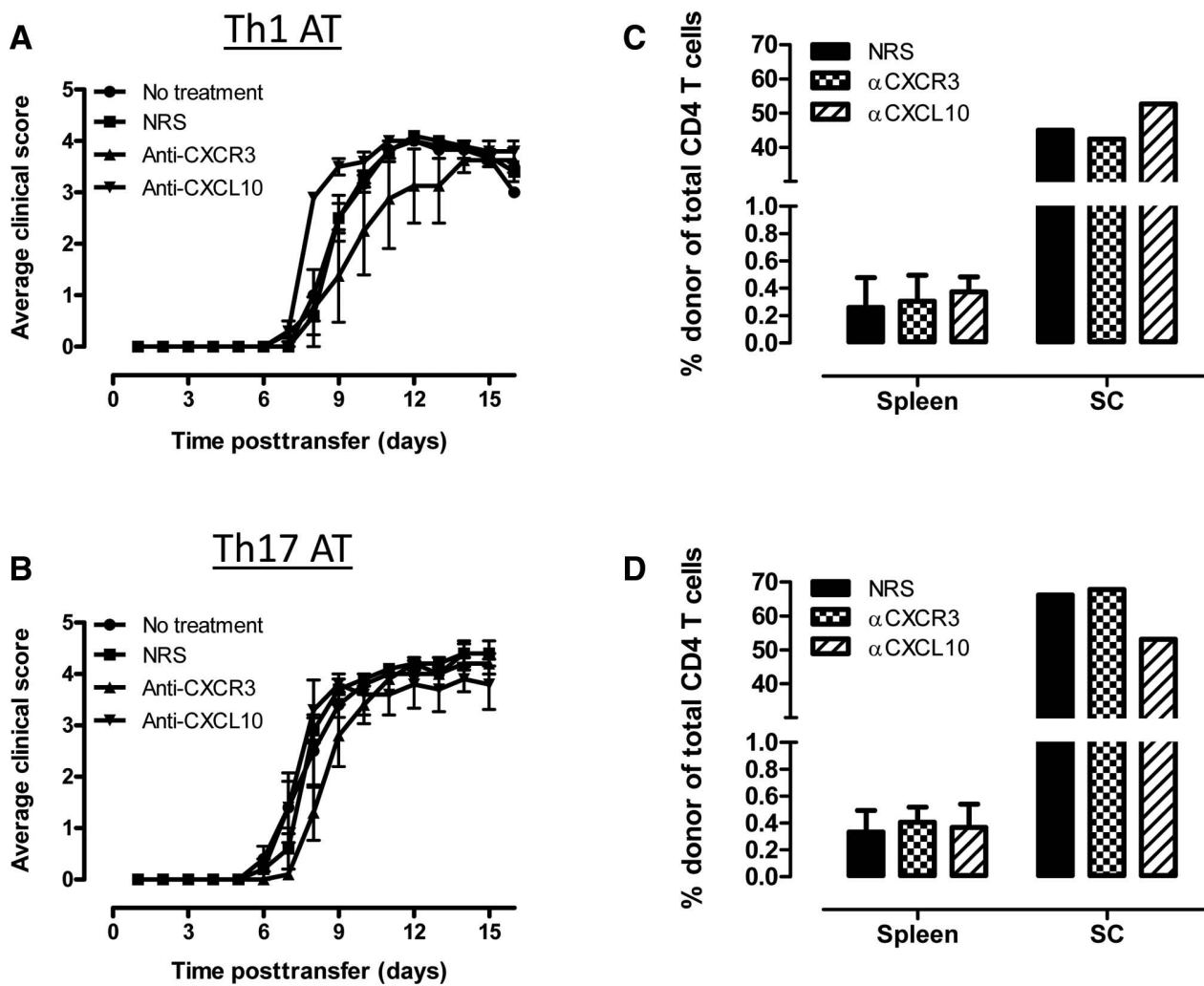

Figure 3. CXCR3 blockade does not attenuate Th1-mediated EAE. (A and B) Naïve WT congenic (CD45.1+) recipients of MOG-specific (A) Th1 or (B) Th17 cells were treated with anti-CXCR3 or anti-CXCL10 antisera, or NRS, every third day from the time of cell transfer to day 15, or not treated at all. Mice were monitored daily for clinical signs of EAE. Data are shown as mean \pm SEM of $n=5$ mice per group from one experiment representative of two performed. (C and D) At the end of the experiment, single-cell suspensions were prepared from spleens (individually) and spinal cords (pooled per group) of (C) Th1 and (D) Th17 recipients for flow cytometric analysis. The isolated cells were stained for extracellular CD4, CD45.1, and CD45.2. The frequency of donor cells among total $\mathrm{CD} 4^{+} \mathrm{T}$ cells is presented as mean $+\mathrm{SEM}$ of $n=5$ mice per group from one experiment representative of two performed.

a clinically relevant setting, neutralizing antisera to CXCR3 or CXCL10, or normal rabbit serum (NRS) was administered to WT mice following the transfer of encephalitogenic T cells. These same reagents, administered at the same dose, have been shown to significantly reduce CNS infiltration by $\mathrm{CD}^{+} \mathrm{T}$ cells in a C57BL/6 mouse model of demyelinating disease induced by mouse hepatitis virus $[27,29]$. Consistent with the results we obtained with knockout mice, neither treatment had a significant impact on the clinical course of EAE, irrespective of the Th lineage of donor $\mathrm{T}$ cells (Fig. 3A and B). The frequency of donor cells among CNSinfiltrating $\mathrm{T}$ cells was similar between adoptive transfer recipients that were treated with NRS or either anti-CXCR3 or anti-CXCL10 antisera (Fig. 3C and D).

\section{Discussion}

The success of natalizumab and fingolimod in suppressing disease activity in individuals with relapsing-remitting MS has validated the strategy of modulating trafficking molecules to attain long-lived clinical remission. However, these agents target adhesion molecules that are widely expressed on leukocytes, thereby increasing the risk of opportunistic infection [30]. Therefore, there is still a need to develop drugs that distinguish between pathogenic and protective leukocytes. Chemokines and their receptors are candidate pharmaceutical targets for disease modification. Variability in the patterns of chemokine receptor expression on Th subsets lends a relatively high degree of selectivity to reagents that disrupt chemokine signaling. Hence, if a chemokine receptor is preferentially expressed on autoimmune effector T cells, administration of a specific antagonist to that receptor may decrease relapse rates with less of an impact on protective immunity than currently available drugs.

A potential drawback of therapies with a restricted mechanism of action is that, despite a favorable safety profile, they might only be effective in a fraction of patients. Indeed, persons with MS comprise a diverse population with regard to clinical course as well as responsiveness to disease-modifying drugs [31]. At present, no clinical features or biomarkers have been identified that reliably predict responsiveness to a particular therapy. Th1 and Th17 effector cells have both been implicated in the development of MS and EAE. Adoptive transfer experiments have shown that these subsets employ distinct adhesion, chemotactic and effector molecules to mediate clinically indistinguishable forms of EAE [23]. In the animal model, such differences in pathogenic mechanisms translate into differential efficacy of specific immunomodulatory 
interventions. Collectively, the above observations suggest that the optimal management of MS will only be realized once strategies are developed to characterize the immune repertoire of individual patients and to customize their therapy accordingly.

The goal of the current study was to assess, as a proof of principle, whether antagonizing a chemokine receptor that is expressed at high levels on a polarized effector T-cell population is a feasible approach for the customized treatment of CNS autoimmunity. CXCR3 is preferentially expressed on encephalitogenic Th1 cells $[13,32,33]$, and on T cells that infiltrate MS and EAE lesions [4-6,9-11], making it a logical therapeutic target for the suppression of Th1-mediated inflammatory demyelinating disease. We found that, even in that special circumstance, blockade of CXCR3, or neutralization of its primary ligand, had no therapeutic impact on the clinical course of EAE. Similarly, $\mathrm{CXCR}^{-/-}$Th1 cells were not compromised in their ability to transfer clinical EAE. In fact, WT recipients of $\mathrm{CXCR}^{-/-}$Th1 cells, or $\mathrm{CXCL} 10^{-/-}$recipients of WT Th1 cells, failed to recover following peak disease to the same extent as their WT counterparts (Fig. 2C and F). It is possible that widespread and diffuse parenchymal distribution of effector cells, as described by Muller et al. in MOG-immunized CXCR3 $^{-/-}$mice [17], results in increased axonal damage and long-term deficits. Of note, administration of a mAb specific for CXCR3 was found to be therapeutically beneficial in a Lewis rat model of EAE induced by the adoptive transfer of unpolarized myelin basic protein reactive $\mathrm{T}$ cells [10]. As in our study, the investigators did not administer Bordetella pertussis toxin (PT) to transfer recipients. The discrepancy between their results and ours further underscores the heterogeneity of encephalitogenic $\mathrm{T}$ cells and reinforces our contention that the importance of a specific molecule as a therapeutic target is context dependent.

Other laboratories have previously reported that Th17 "sentinel" cells traverse the blood-brain barrier at the inception of EAE and release vasoactive substances that permit the subsequent infiltration of Th1 cells $[26,34,35]$. This raises the possibility that in our experimental paradigms, neuroinflammation is initiated by a minor subpopulation of Th17 contaminants within the pool of IL-12-polarized donor cells. We deem this unlikely since we were unable to detect $\mathrm{IL}_{-1} 7^{+}$cells among IL-12-polarized donor T cells. Furthermore, we did not detect ROR $\gamma \mathrm{t}$ transcripts in mRNA extracted from donor cells immediately prior to adoptive transfer (data not shown). It has also been reported that CNS expression of ELR $^{-}$CXC chemokines leads to the local accumulation of CXCR3 ${ }^{+}$ Tregs $[17,36]$. By extension, mice with a disrupted CXCR3/CXC chemokine pathway could be relatively susceptible to EAE due to a dearth of Tregs in target organ infiltrates. However, we found no difference in the percentage of FoxP3 ${ }^{+} \mathrm{T}$ cells in the CNS of WT and CXCL10 $0^{-/-}$hosts with Th1-mediated EAE. Similarly, FoxP3 ${ }^{+}$ donor cells occurred at the same frequency in the adoptive recipients of CXCR3 ${ }^{-1-}$ and WT Th1-polarized cells (data not shown).

We believe that the most likely explanation for the dispensability of CXCR3/CXC chemokine interactions in the manifestation of Th1-mediated EAE lies in the complexity of chemokine pathways that arise at sites of neuroinflammation. Th1 cells express CCR 2 and CCR5 [33,37], and their respective ligands, CCL2 and
CCL5, are upregulated in the CNS during EAE [38, 39]. Analogous to our findings with $\mathrm{CXCR}^{-/-}$mice, $\mathrm{CCR} 2^{-/-}$and $\mathrm{CCR}^{-/-}$ mice remain susceptible to $\mathrm{EAE}[40,41]$. Whether this is due to the adoption of compensatory trafficking pathways by the single knockout mice will only be determined by future experiments with double or triple knockouts. The redundancy of chemokines in the EAE model is further illustrated by a previous publication showing that simultaneous blockade of CXCR3 and CXCR4 was therapeutically efficacious in adoptively transferred EAE in comparison to targeting CXCR3 alone [42].

In conclusion, the strategy of antagonizing individual chemokine/chemokine receptor interactions in individuals with MS, including those patients with a skewed effector population, might be undermined by inherent redundancies in chemokine networks. The ideal therapeutic target would be a molecule that is exclusively expressed on autoimmune effector cells and that is critical for pathogenicity. Until such a molecule is identified, the treatment of autoimmune disease will have to balance therapeutic effectiveness against the untoward consequences of immunosuppression.

\section{Materials and methods}

\section{Mice}

About 8- to 12-week-old C57BL/6 and CD45.1 congenic B6 Ly5.2/Cr mice were obtained from NCI Frederick (Frederick, MD, USA). $\mathrm{CxCr}^{-/-}$mice were provided by C. Gerard, the generation and characterization of which were described previously [43]. CXCL10 ${ }^{-/-}$mice were obtained from The Jackson Laboratory (Bar Harbor, ME, USA). Mice were housed in micro-isolator cages under specific pathogen-free, barrier facility conditions. All procedures were conducted in strict accordance with protocols approved by the University of Michigan Committee on Use and Care of Animals.

\section{Induction and treatment of EAE}

Active induction of EAE involved s.c. injection of $100 \mu \mathrm{g} \mathrm{MOG}_{35-55}$ MEVGWYRSP-FSRVVHLYRNGK (Biosynthesis, Lewisville, TX, USA) in CFA (Difco, Detroit, MI, USA) containing $4 \mathrm{mg} / \mathrm{mL}$ heatkilled Mycobacterium tuberculosis H37Ra (Difco). Each mouse also received $300 \mathrm{ng}$ of Bordetella PT(List Biological Laboratories) i.p. on day 0 and 2 postimmunization. For passive induction, mice were immunized as above, but without administration of PT. Ten days postimmunization, a single-cell suspension was prepared from pooled draining inguinal, axillary, and brachial LNs and passed through a $70 \mu \mathrm{m}$ cell strainer (BD Falcon, Franklin Lakes, NJ, USA). LN cells were cultured in vitro for 4 days with MOG $_{35-55}$ under conditions favorable to the generation of Th1 cells (rmIL-12, $6 \mathrm{ng} / \mathrm{mL}$; rmIFN- $\gamma, 2 \mathrm{ng} / \mathrm{mL}$; anti-IL-4 (clone 11B11), $10 \mu \mathrm{g} / \mathrm{mL}$ ) or Th17 cells (rmIL- $1 \alpha, 10 \mathrm{ng} / \mathrm{mL}$; rmIL-23, $8 \mathrm{ng} / \mathrm{mL}$; 
anti-IL-4 (clone 11B11), $10 \mu \mathrm{g} / \mathrm{mL}$; anti-IFN- $\gamma$ (clone XMG1.2) $10 \mu \mathrm{g} / \mathrm{mL}$ ). After 4 days culture, LN cells were collected and $2 \times$ $10^{6} \mathrm{CD}^{+}{ }^{+} \mathrm{T}$ cells injected i.p. in sterile PBS. Mice were observed daily for signs of EAE and the severity of neurological impairment rated as follows: 0 , no discernible deficit; 1 , limp tail; 2 , impaired gait and/or ability to flip over from a supine position; 3, partial hindlimb paralysis; 4, total hindlimb paralysis; 5 , moribund.

Rabbit polyclonal antisera specific for mouse CXCR3 and CXCL10 were provided by Dr. Thomas Lane, the generation of which has previously been described [44]. These reagents have been shown to be specific for mCXCR3 and mCXCL10 and do not cross-react with a panel of other human and murine recombinant cytokines $[27,29,44]$. They have been shown to block CD4 ${ }^{+}$T-cell infiltration in vivo $[27,29,44]$. Experimental groups of mice were injected i.p. with $0.5 \mathrm{~mL}$ anti-mCXCR3 or anti-mCXCL10 every third day from $\mathrm{d} 0$ to $\mathrm{d} 15$ post-T-cell transfer. NRS from the same preinoculated rabbits was used as a control.

\section{ELISPOT assays}

Antigen-specific cytokine production was determined in spleen and dLN cells and mononuclear cells isolated by Percoll density centrifugation from the pooled SCs of mice perfused with PBS, following culture for $24 \mathrm{~h}$ in 96-well filtration plates (Millipore), with or without $50 \mu \mathrm{g} / \mathrm{mL} \mathrm{MOG}_{35-55}$. Antibodies from eBioscience were anti-IL-17 (TC11-18H10), biotinylated anti-IL-17 (TC118H4), IFN- $\gamma$ (AN18), and biotinylated anti-IFN- $\gamma$ (R4-6A2). Streptavidin-alkaline phosphatase (Southern Biotech) and an alkaline phosphatase substrate kit (Vector Laboratories) were used to identify trapped cytokine. Spots were counted using the CTL ImmunoSpot Analyzer (Cellular Technology) with ImmunoSpot software, and the number of spots in the medium only wells subtracted.

\section{Real-time RT-PCR}

RNA was harvested from whole SC using the Trizol (Invitrogen)/chloroform method followed by RT into cDNA using the High-Capacity cDNA Reverse Transcription Kit (Applied Biosystems). Primers and probes were designed using Beacon Designer and synthesized by Integrated DNA Technologies. Samples were analyzed on an iCycler PCR machine (Bio-Rad Laboratories). Data were normalized to the endogenous control $\beta$-actin and expressed as fold increase over SCs from naïve mice.

\section{Intracellular cytokine staining and flow cytometry}

Splenocytes, mononuclear cells isolated by Percoll density centrifugation from the pooled SCs of mice perfused with PBS, or polarized dLN cells following culture were activated $(2 \times$ $10^{6}$ cells/mL) with PMA (50 ng/mL; Sigma) and ionomycin (2 $\mu \mathrm{g} / \mathrm{mL}$; Sigma), in the presence of brefeldin A $(5 \mu \mathrm{g} / \mathrm{mL})$, for $6 \mathrm{~h}$ at $37^{\circ} \mathrm{C}$. Cells were washed and blocked with Fc block (clone 2.4G2; $50 \mu \mathrm{g} / \mathrm{mL}$ ) before extracellular staining with fluorochrome-conjugated antibodies for CD3, CD4, CD45.1, and CD45.2 (eBioscience). Cells were then fixed with 4\% paraformaldehyde, permeabilized with saponin (Sigma), and stained intracellularly with fluorochrome-conjugated antibodies for IL-17 or IFN- $\gamma$ (eBioscience). Flow cytometric analysis was performed using a FACSCanto II flow cytometer (Becton-Dickinson) and analyzed with FloJo software (Tree Star, Inc.), with gating set on isotype controls. Absolute numbers of donor and host cells were determined by multiplying the total cell count per tissue by the frequency of congenic cells among the total $\mathrm{CD}^{+}{ }^{+} \mathrm{T}$-cell population, within the live cell gate.

\section{Statistical analysis}

Statistical analyses were performed using GraphPad Prism statistical analysis software. Group differences were analyzed by unpaired, two-tailed Student's $t$-test, while a two-way ANOVA with repeated measures was applied when comparing different experimental groups over time. $p$-values of 0.05 or less were considered significant.

Acknowledgements: The authors thank Dr. Thomas Lane for providing antisera to CXCR3 and CXCL10. We also thank Dr. Julie Rumble and Dr. Nick Lukacs for critical reading of the manuscript. This work was supported by the National Multiple Sclerosis Society Grant CA 1037A (B.M.S) and by the National Multiple Sclerosis Society Grant FG 1985-A-1 (S.J.L.).

Conflict of interest: The authors declare no financial or commercial conflict of interest.

\section{References}

1 Compston, A. and Coles, A., Multiple sclerosis. Lancet 2008. 372: 1502 1517.

2 Polman, C. H., O'Connor, P. W., Havrdova, E., Hutchinson, M., Kappos, L., Miller, D. H., Phillips, J. T. et al., A randomized, placebo-controlled trial of natalizumab for relapsing multiple sclerosis. N. Engl. J. Med. 2006. 354: 899-910.

3 Kappos, L., Radue, E. W., O'Connor, P., Polman, C., Hohlfeld, R., Calabresi, P., Selmaj, K. et al., A placebo-controlled trial of oral fingolimod in relapsing multiple sclerosis. N. Engl. J. Med. 2010. 362: 387-401.

4 Balashov, K. E., Rottman, J. B., Weiner, H. L. and Hancock, W. W., CCR5(+) and CXCR3(+) T cells are increased in multiple sclerosis and their ligands MIP-1alpha and IP-10 are expressed in demyelinating brain lesions. Proc. Natl. Acad. Sci. USA 1999. 96: 6873-6878. 
5 Simpson, J. E., Newcombe, J., Cuzner, M. L. and Woodroofe, M. N., Expression of the interferon-gamma-inducible chemokines IP-10 and Mig and their receptor, CXCR3, in multiple sclerosis lesions. Neuropathol. Appl. Neurobiol. 2000. 26: 133-142.

6 Sorensen, T. L., Tani, M., Jensen, J., Pierce, V., Lucchinetti, C., Folcik, V. A., Qin, S. et al., Expression of specific chemokines and chemokine receptors in the central nervous system of multiple sclerosis patients. J. Clin. Invest. 1999. 103: 807-815.

7 Sorensen, T. L., Trebst, C., Kivisakk, P., Klaege, K. L., Majmudar, A., Ravid, R., Lassmann, H. et al., Multiple sclerosis: a study of CXCL10 and CXCR3 co-localization in the inflamed central nervous system. J. Neuroimmunol. 2002. 127: 59-68.

8 Ransohoff, R. M., Hamilton, T. A., Tani, M., Stoler, M. H., Shick, H. E., Major, J. A., Estes, M. L. et al., Astrocyte expression of mRNA encoding cytokines IP-10 and JE/MCP-1 in experimental autoimmune encephalomyelitis. FASEB J. 1993. 7: 592-600.

9 O'Connor, R. A., Floess, S., Huehn, J., Jones, S. A. and Anderton, S. M., Foxp3(+) Treg cells in the inflamed CNS are insensitive to IL-6-driven IL-17 production. Eur. J. Immunol. 2012. 42: 1174-1179.

10 Sporici, R. and Issekutz, T. B., CXCR3 blockade inhibits T-cell migration into the CNS during EAE and prevents development of adoptively transferred, but not actively induced, disease. Eur. J. Immunol. 2010. 40: 2751-2761.

11 O'Connor, R. A., Li, X., Blumerman, S., Anderton, S. M., Noelle, R. J. and Dalton, D. K., Adjuvant immunotherapy of experimental autoimmune encephalomyelitis: immature myeloid cells expressing CXCL10 and CXCL16 attract CXCR3+CXCR6+ and myelin-specific T cells to the draining lymph nodes rather than the central nervous system. J. Immunol. 2012. 188: 2093-2101.

12 Loetscher, M., Loetscher, P., Brass, N., Meese, E. and Moser, B., Lymphocyte-specific chemokine receptor CXCR3: regulation, chemokine binding and gene localization. Eur. J. Immunol. 1998. 28: 3696-3705.

13 Lord, G. M., Rao, R. M., Choe, H., Sullivan, B. M., Lichtman, A. H., Luscinskas, F. W. and Glimcher, L. H., T-bet is required for optimal proinflammatory CD4+ T-cell trafficking. Blood 2005. 106: 3432-3439.

14 Nakajima, C., Mukai, T., Yamaguchi, N., Morimoto, Y., Park, W. R., Iwasaki, M., Gao, P. et al., Induction of the chemokine receptor CXCR3 on TCR-stimulated T cells: dependence on the release from persistent TCRtriggering and requirement for IFN-gamma stimulation. Eur. J. Immunol. 2002. 32: 1792-1801.

15 Klein, R. S., Izikson, L., Means, T., Gibson, H. D., Lin, E., Sobel, R. A., Weiner, H. L. et al., IFN-inducible protein 10/CXC chemokine ligand 10independent induction of experimental autoimmune encephalomyelitis. J. Immunol. 2004. 172: 550-559.

16 Liu, L., Huang, D., Matsui, M., He, T. T., Hu, T., Demartino, J., Lu, B. et al., Severe disease, unaltered leukocyte migration, and reduced IFNgamma production in CXCR3-/- mice with experimental autoimmune encephalomyelitis. J. Immunol. 2006. 176: 4399-4409.

17 Muller, M., Carter, S. L., Hofer, M. J., Manders, P., Getts, D. R., Getts, M. T., Dreykluft, A. et al., CXCR3 signaling reduces the severity of experimental autoimmune encephalomyelitis by controlling the parenchymal distribution of effector and regulatory $\mathrm{T}$ cells in the central nervous system. J. Immunol. 2007. 179: 2774-2786.

18 Byrne, F. R., Winters, A., Brankow, D., Hu, S., Juan, T., Steavenson, S., Doellgast, G. et al., An antibody to IP-10 is a potent antagonist of cell migration in vitro and in vivo and does not affect disease in several animal models of inflammation. Autoimmunity 2009. 42: 171-182.

19 Narumi, S., Kaburaki, T., Yoneyama, H., Iwamura, H., Kobayashi, Y. and Matsushima, K., Neutralization of IFN-inducible protein 10/CXCL10 exac- erbates experimental autoimmune encephalomyelitis. Eur. J. Immunol. 2002. 32: 1784-1791.

20 Lalor, S. J., Dungan, L. S., Sutton, C. E., Basdeo, S. A., Fletcher, J. M. and Mills, K. H., Caspase-1-processed cytokines IL-1beta and IL-18 promote IL-17 production by gammadelta and CD4 T cells that mediate autoimmunity. J. Immunol. 2011. 186: 5738-5748.

21 Durelli, L., Conti, L., Clerico, M., Boselli, D., Contessa, G., Ripellino, P., Ferrero, B. et al., T-helper 17 cells expand in multiple sclerosis and are inhibited by interferon-beta. Ann. Neurol. 2009. 65: 499-509.

22 Kebir, H., Ifergan, I., Alvarez, J. I., Bernard, M., Poirier, J., Arbour, N., Duquette, P. et al., Preferential recruitment of interferon-gammaexpressing TH17 cells in multiple sclerosis. Ann. Neurol. 2009. 66: 390-402.

23 Kroenke, M. A., Carlson, T. J., Andjelkovic, A. V. and Segal, B. M., IL12- and IL-23-modulated $\mathrm{T}$ cells induce distinct types of EAE based on histology, CNS chemokine profile, and response to cytokine inhibition. J. Exp. Med. 2008. 205: 1535-1541.

24 Mehling, M., Lindberg, R., Raulf, F., Kuhle, J., Hess, C., Kappos, L. and Brinkmann, V., Th17 central memory T cells are reduced by FTY720 in patients with multiple sclerosis. Neurology 2010. 75: 403-410.

25 Ramgolam, V., Sha, Y., Jin, J., Zhang, X. and Markovic-Plese, S., IFNbeta inhibits human Th17 cell differentiation. J. Immunol. 2009. 183: 5418-5427.

26 Reboldi, A., Coisne, C., Baumjohann, D., Benvenuto, F., Bottinelli, D., Lira, S., Uccelli, A. et al., C-C chemokine receptor 6-regulated entry of TH-17 cells into the CNS through the choroid plexus is required for the initiation of EAE. Nat. Immunol. 2009. 10: 514-523.

27 Stiles, L. N., Hosking, M. P., Edwards, R. A., Strieter, R. M. and Lane, T. E., Differential roles for CXCR3 in CD4+ and CD8+ T cell trafficking following viral infection of the CNS. Eur. J. Immunol. 2006. 36: 613-622.

28 Hirota, K., Duarte, J. H., Veldhoen, M., Hornsby, E., Li, Y., Cua, D. J., Ahlfors, H. et al., Fate mapping of IL-17-producing T cells in inflammatory responses. Nat. Immunol. 2011. 12: 255-263.

29 Stiles, L. N., Liu, M. T., Kane, J. A. and Lane, T. E., CXCL10 and trafficking of virus-specific $\mathrm{T}$ cells during coronavirus-induced demyelination. Autoimmunity 2009. 42: 484-491.

30 Berger, J. R. and Houff, S., Opportunistic infections and other risks with newer multiple sclerosis therapies. Ann. Neurol. 2009. 65: 367-377.

31 Rudick, R. A. and Polman, C. H., Current approaches to the identification and management of breakthrough disease in patients with multiple sclerosis. Lancet Neurol. 2009. 8: 545-559.

32 Bonecchi, R., Bianchi, G., Bordignon, P. P., D’Ambrosio, D., Lang, R., Borsatti, A., Sozzani, S. et al., Differential expression of chemokine receptors and chemotactic responsiveness of type $1 \mathrm{~T}$ helper cells (Th1s) and Th2s. J. Exp. Med. 1998. 187: 129-134.

33 Sallusto, F., Lanzavecchia, A. and Mackay, C. R., Chemokines and chemokine receptors in T-cell priming and Th1/Th2-mediated responses. Immunol. Today 1998. 19: 568-574.

34 Kebir, H., Kreymborg, K., Ifergan, I., Dodelet-Devillers, A., Cayrol, R., Bernard, M., Giuliani, F. et al., Human TH17 lymphocytes promote bloodbrain barrier disruption and central nervous system inflammation. Nat. Med. 2007. 13: 1173-1175.

35 Lees, J., Iwakura, Y. and Russell, J., Host T cells are the main producers of IL-17 within the central nervous system during initiation of experimental autoimmune encephalomyelitis induced by adoptive transfer of Th1 cell lines. J. Immunol. 2008. 180: 8066-8072.

36 Koch, M. A., Tucker-Heard, G., Perdue, N. R., Killebrew, J. R., Urdahl, K. B. and Campbell, D. J., The transcription factor T-bet controls regulatory $\mathrm{T}$ cell homeostasis and function during type 1 inflammation. Nat. Immunol. 2009. 10: 595-602. 
37 Sato, W., Tomita, A., Ichikawa, D., Lin, Y., Kishida, H., Miyake, S., Ogawa, M. et al., CCR2(+)CCR5(+) T cells produce matrix metalloproteinase-9 and osteopontin in the pathogenesis of multiple sclerosis. J. Immunol. 2012. 189: 5057-5065.

38 Glabinski, A. R., Tani, M., Tuohy, V. K., Tuthill, R. J. and Ransohoff, R. M., Central nervous system chemokine mRNA accumulation follows initial leukocyte entry at the onset of acute murine experimental autoimmune encephalomyelitis. Brain Behav. Immun. 1995. 9: 315-330.

39 Glabinski, A. R., Tuohy, V. K. and Ransohoff, R. M., Expression of chemokines RANTES, MIP-1alpha and GRO-alpha correlates with inflammation in acute experimental autoimmune encephalomyelitis. Neuroimmunomodulation 1998. 5: 166-171.

40 Tran, E. H., Kuziel, W. A. and Owens, T., Induction of experimental autoimmune encephalomyelitis in C57BL/6 mice deficient in either the chemokine macrophage inflammatory protein-1alpha or its CCR5 receptor. Eur. J. Immunol. 2000. 30: 1410-1415.

41 Gaupp, S., Pitt, D., Kuziel, W. A., Cannella, B. and Raine, C. S., Experimental autoimmune encephalomyelitis (EAE) in CCR2(-/-) mice: susceptibility in multiple strains. Am. J. Pathol. 2003. 162: 139-150.

42 Kohler, R. E., Comerford, I., Townley, S., Haylock-Jacobs, S., ClarkLewis, I. and McColl, S. R., Antagonism of the chemokine receptors CXCR3 and CXCR4 reduces the pathology of experimental autoimmune encephalomyelitis. Brain Pathol. 2008. 18: 504-516.
43 Hancock, W. W., Lu, B., Gao, W., Csizmadia, V., Faia, K., King, J. A., Smiley, S. T. et al., Requirement of the chemokine receptor CXCR3 for acute allograft rejection. J. Exp. Med. 2000. 192: 1515-1520.

44 Belperio, J. A., Keane, M. P., Burdick, M. D., Lynch, J. P., 3rd, Xue, Y. Y., Li, K., Ross, D. J. et al., Critical role for CXCR3 chemokine biology in the pathogenesis of bronchiolitis obliterans syndrome. J. Immunol. 2002. 169: 1037-1049.

Abbreviations: dLN: draining LN - ELR: glutamic acid-leucine-arginine motif · MOG: myelin oligodendrocyte glycoprotein · NRS: normal rabbit serum · PT: pertussis toxin - SC: spinal cord

Full correspondence: Dr. Benjamin M. Segal, Department of Neurology, Holtom-Garrett Program in Neuroimmunology, University of Michigan, 4013 BSRB, 109 Zina Pitcher Place, SPC 2200, Ann Arbor, MI 48109-2200, USA

Fax: +1-734-615-7300

e-mail: bmsegal@med.umich.edu

Received: 4/3/2013

Revised: 5/6/2013

Accepted: 16/7/2013

Accepted article online: 22/7/2013 